\title{
THE ORBITAL PERIOD OF THE ACCRETING PULSAR GX $1+4$
}

\author{
M. G. Pereira, J. Braga, and F. Jablonski \\ Divisão de Astrofísica, Instituto Nacional de Pesquisas Espaciais, CP 515, 12201-970, São José dos Campos, \\ Brazil; marildo@das.inpe.br, braga@das.inpe.br, chico@das.inpe.br \\ Received 1999 April 27; accepted 1999 October 6; published 1999 October 29
}

\begin{abstract}
We report strong evidence for a $\sim 304$ day periodicity in the spin history of the accretion-powered pulsar GX $1+4$ that is most probably associated with the orbital period of the system. We have used data from the Burst and Transient Source Experiment on the Compton Gamma-Ray Observatory to show a clear periodic modulation of the pulsar frequency from 1991 to date, in excellent agreement with the ephemeris proposed by Cutler, Dennis, \& Dolan in 1986. Our results indicate that the orbital period of GX $1+4$ is $303.8 \pm 1.1$ days, making it the widest known low-mass X-ray binary system by more than 1 order of magnitude and putting this long-standing question to rest. A likely scenario for this system is an elliptical orbit in which the neutron star decreases its spin-down rate (or even exhibits a momentary spin-up behavior) at periastron passages due to the higher torque exerted by the accretion disk onto the magnetosphere of the neutron star. These results are not inconsistent with either the X-ray pulsed flux light curve measured by BATSE during the same epoch or the X-ray flux history from the All-Sky Monitor on board the Rossi X-Ray Timing Explorer.
\end{abstract}

Subject headings: accretion, accretion disks — binaries: symbiotic — pulsars: individual (GX 1+4) stars: neutron $-\mathrm{X}$-rays: stars

\section{INTRODUCTION}

GX 1+4 is a bright Galactic center accretion-powered pulsar in a low-mass X-ray binary system (LMXB) discovered in the early 1970s (Lewin, Ricker, \& McClintock 1971). Throughout the 1970s the pulsar exhibited a spin-up behavior with the pulsation period decreasing from $135 \mathrm{~s}$ to less than $110 \mathrm{~s}$ (Cutler, Dennis, \& Dolan 1986, hereafter CDD86, and references therein), corresponding to a spin-up rate of $\dot{P} \sim-2 \mathrm{~s} \mathrm{yr}^{-1}$. After experiencing an extended low-intensity state in the early $1980 \mathrm{~s}$ (Hall \& Develaar 1983; McClintock \& Leventhal 1989), GX $1+4$ reemerged in a spin-down state (Makishima et al. 1988; Sakao et al. 1990) with approximately the same $|\dot{P}|$ and has stayed in this state ever since, with occasional short-term variations of $\dot{P}$.

Infrared observations and optical spectroscopy of GX $1+4$ established a rare association of a neutron star with a M5 III giant star, V2116 Oph, in a symbiotic binary system (Glass \& Feast 1973; Davidsen, Malina, \& Bowyer 1977; Chakrabarty \& Roche 1997). The identification was made secure by a ROSAT accurate position determination (Predehl, Friedrich, \& Staubert 1995) and by the discovery of optical pulsations in V2116 Oph consistent with the spin period of the neutron star (Jablonski et al. 1997; Pereira et al. 1997). In comparison with the other four known LMXB accretion-powered pulsars (GRO J1744-28, Her X-1, 4U 1627-67, and the recently discovered millisecond accreting pulsar SAX J1808.4-3658; Wijnands \& van der Klis 1998), GX 1+4 has a much longer (factor of 100) spin period, and its orbital period, albeit not securely measured until this work, was known to be at least 1 order of magnitude longer than the periods of the other systems. Physically quantitative lower limits on the binary period of GX $1+4$ were derived by Chakrabarty \& Roche (1997), who showed that the binary period must be at least 100 days and is probably more than 260 days.

In 1991, the Burst and Transient Source Experiment (BATSE) on the Compton Gamma-Ray Observatory (CGRO) initiated a continuous and nearly uniform monitoring of GX $1+4$. The BATSE observations confirmed the spin-down trend with occasional dramatic spin-up/spin-down torque reversal events (Chakrabarty 1996; Chakrabarty et al. 1997; Nelson et al. 1997).

Attempts to find the orbital period of GX $1+4$ by Doppler shifts of the pulsar pulse timing or optical lines have both been inconclusive so far. For the X-ray timing measurements, the accretion torque magnitude is much larger than the expected orbital Doppler shifts, and the torque fluctuations have significant power at the timescales comparable to the expected binary period (Chakrabarty 1996). In the case of the optical lines, the

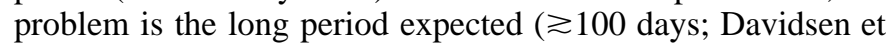
al. 1977; Doty, Hoffman, \& Lewin 1981; Sood et al. 1995). Long-term optical photometry in the $R$ band has shown variations in the light curve with periods of $\sim 30$ and $\sim 110$ days (Pereira, Braga, \& Jablonski 1996; Pereira 1998). Using a small number of X-ray measurements carried out during the spin-up phase of GX 1+4 in the 1970s, CDD86 produced an ephemeris for predicting periodical enhancements in the spin-up rate of the neutron star. A possible interpretation for this periodic behavior is that the neutron star and the red giant are in an elliptical orbit with a 304 day period.

In this work, we report the results of a comprehensive timeseries analysis of the BATSE data on GX $1+4$ in an attempt to find the orbital period of the system. We discuss the implications that can be drawn from our results in light of the possible models for this source and show that the elliptical orbit interpretation is probably the correct one. We present a refined version of the ephemeris originally proposed by CDD86.

\section{DATA ANALYSIS AND RESULTS}

The frequency and the pulsed flux data between JD 2,448,376.5 and 2,451,138.5 (i.e., 1991 April 29 to 1998 October 20) used in this work were obtained from Chakrabarty (1996) and from the BATSE public domain data. ${ }^{1}$ The $20-50$ $\mathrm{keV}$ pulsed signals are extracted from DISCLA $1.024 \mathrm{~s}$ chan-

\footnotetext{
${ }^{1}$ The BATSE public domain data are available at http://gammaray.msfc nasa.gov/batse/pulsar.
} 


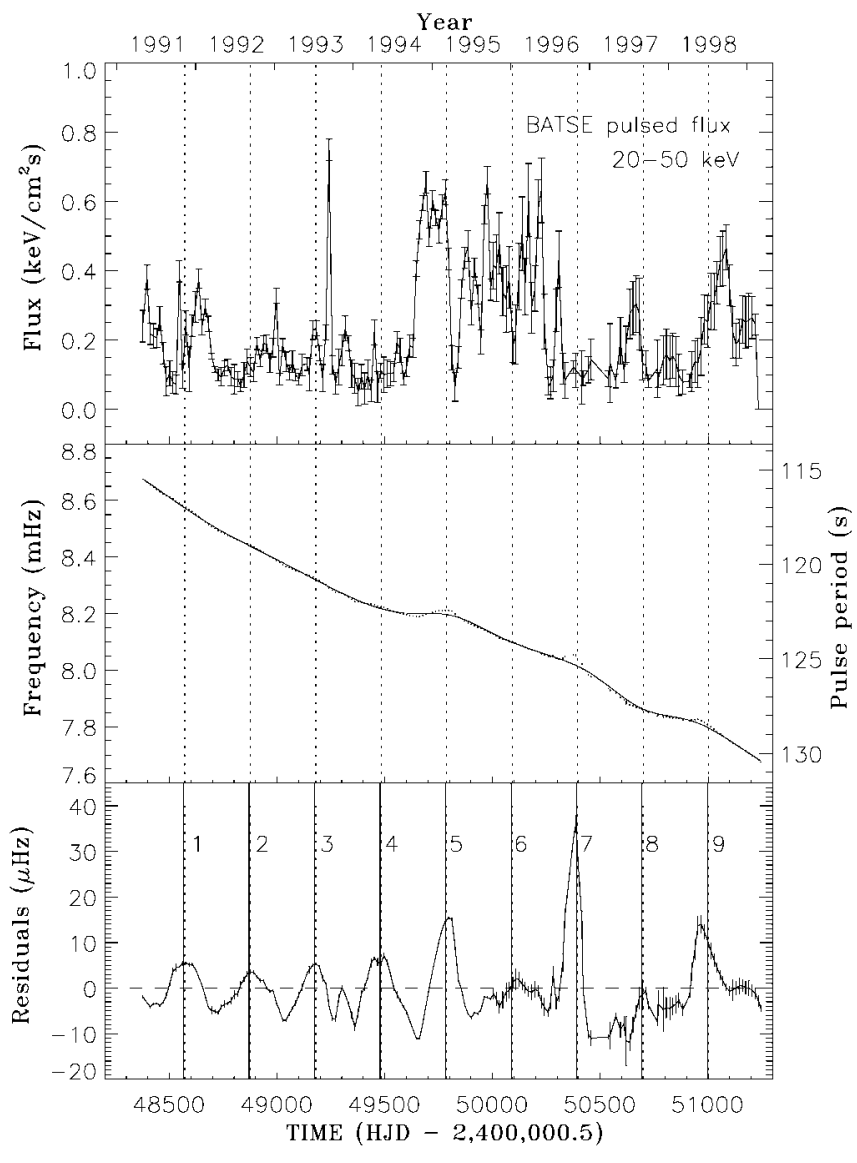

FIG. 1.-Top: Light curve of the $20-50 \mathrm{keV}$ pulsed flux of GX $1+4$ as measured by BATSE from 1991 to 1998. Middle: GX 1+4 frequency measurements by BATSE over the same period. The error bars are in general smaller than the size of the dots. The solid curve is a cubic spline fit to the data. Bottom: Frequency residuals. The dotted vertical lines mark the times predicted by the ephemeris calculated in this work, whereas the solid vertical lines show the predictions according to CDD86's ephemeris. The events of positive residual frequency modulation are labeled for reference in the text.

nel 1 data. After being weighted according to the aspect angle of each detector to a source on an existing source list, data are treated to remove the background variations, barycentered, and epoch-folded over a set of grid points in the vicinity of the expected pulse frequency. Frequency and modeled flux are inspected daily for significant detections, and the reports are produced twice a week. Fifteen day mean values for the fluxes and pulse frequencies of GX $1+4$ were calculated for the entire data set.

A data set of GX $1+4$ residual pulsation frequencies was obtained from the frequency history by subtraction of a standard cubic spline function to remove low-frequency variations in the spin-down trend. The fitting points are mean frequency values calculated over suitably chosen time intervals. The results of the spline fitting are fairly insensitive to intervals greater than $\sim 200$ days between fitting points (we have used $\Delta t=215$ days). The pulsed X-ray flux, frequency history, and residual frequencies are shown in Figure 1 as functions of time.

We have carried out a power spectrum analysis to search for periodicities of less than 1000 days in both the residual frequency and the pulsed flux data. A Lomb-Scargle periodogram (Press et al. 1992), suitable for time series with gaps, shows a significant periodic signal at 302.0 days (Fig. 2) in the residual frequency time series. This value is insensitive to any over-

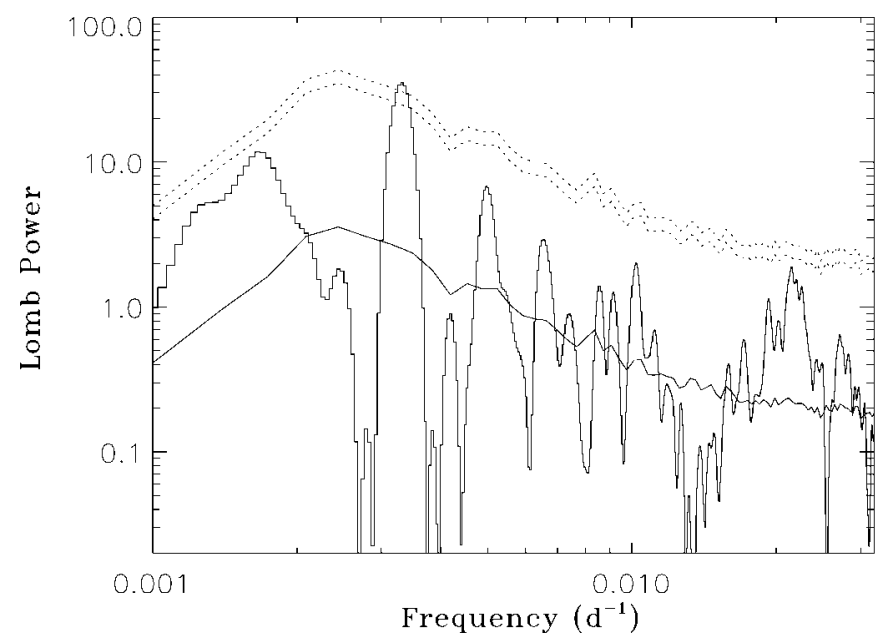

FIG. 2.-Lomb-Scargle periodogram of the frequency residuals of GX $1+4$ from 1991 to 1998, represented by the histogram-type solid line. The standard solid line is the mean of 1500 numerical simulations carried out in order to calculate the significance level of the detection. The upper dotted line indicates a significance level of 0.001 , whereas the lower dotted line indicates a significance level of 0.01 .

sampling factor greater than 2 over the Nyquist interval. The power spectrum shows a red noise with an approximate powerlaw index of -2 . In order to estimate the statistical significance of the detection, a series of numerical simulations of the frequency time series with $1 \sigma$ Gaussian deviations (using the error bars of the data points) were performed. In the frequency domain of the simulated light curves, we forced the power spectra to have a power-law index of -2 and transformed the results back to the time domain. We then selected the times in the simulated light curves to match the observed times for the data points and subtracted the spline with $\Delta t=215$ days. We finally calculated the Lomb periodogram of the simulated time series. The simulations show that the use of the 215 day spline, besides providing an effective filter for frequencies below $\sim 2 \times 10^{-3}$ day $^{-1}$, does not produce power in any specific frequency in the range of interest, i.e., no monochromatic or even QPO signals are produced. By comparing the amplitude of our 302 day peak with the local value obtained by the mean of the numerical simulations (the peak is a factor of 13.91 higher), we obtain a statistical significance of $99.98 \%$ for the detection. Epoch-folding the data using the 302 day period yields a $1 \sigma$ uncertainty of 1.7 days. In the pulsed flux data, the most interesting feature is a wide structure of low-significance peaks observed in the range 200-500 days, with no significant peak at $\sim 300$ days.

By analyzing the variation of the period of GX $1+4$ during the spin-up phase in the 1970s, CDD86 proposed a 304 day orbital period and an ephemeris to predict the events of enhanced spin-up: $T=\mathrm{JD} 2,444,574.5 \pm 304 n$, where $n$ is an integer. This ephemeris is based on four events discussed by the authors, whose existence was inferred from ad hoc assumptions and extrapolations of the observations. The projected enhanced spin-up events derived from that ephemeris for the epochs contained in the BATSE data set (solid vertical lines, Fig. 1, bottom) are in excellent agreement with the BATSE reduced spin-down and spin-up events. The BATSE data set is obviously significantly more reliable than CDD86's inasmuch as it is based on nine well-covered events measured with the same instrument as opposed to the four events discussed 
in CDD86. The striking agreement of CDD86's ephemeris with the BATSE observations is very conspicuous and gives very strong support to the claim that the orbital period of the system is indeed $\sim 304$ days. Taking integer cycle numbers, with the $T 0$ epoch of CDD86 as cycle -23 and performing a linear least-squares fit to the frequency residuals seen in the lower panel of Figure 1, we find that the following ephemeris can represent the time of occurrence $T$ of the maxima in the frequency residuals:

$$
T=\mathrm{JD} 2,448,571.3( \pm 3.2) \pm 303.8( \pm 1.1) n,
$$

where $n$ is any integer. The events predicted by the above ephemeris are shown as vertical dashed lines in the three panels of Figure 1. Taking into account a conservative uncertainty estimate of 30 days for the peaks of the BATSE events, the reduced $\chi^{2}$ of the fit is $\chi_{r}^{2}=0.61$. The value of $303.8 \pm 1.1$ days for the orbital period is consistent with the one obtained through power spectrum analysis performed on the BATSE data, which gives further support for the period determination.

\section{DISCUSSION}

In the BATSE era, the long-term frequency history of GX $1+4$ shown in Figure 1 (middle) exhibits a characteristic spin-down trend with an average rate of $\sim 1.8 \mathrm{~s} \mathrm{yr}^{-1}$. Frequency derivative reversals occur on times preceding the epochs of events labeled 5, 7, and 9 in the bottom panel. The upper panel of Figure 1 shows that these events are somewhat correlated with rather intense flares in the pulsed flux. In the 1970s, when the measurements used by CDD86 were carried out, the source was in a spin-up extended state. The scenario proposed by CDD86 to explain the periodic occurrence of enhanced spinup events was that the system was in an elliptical orbit and the periastron passages would occur when $\dot{P}$ is maximum, as expected in standard accretion from a spherically expanding stellar wind. It is widely accepted today, as inferred from GX 1+4's optical/IR properties (Jablonski et al. 1997; Chakrabarty, van Kerkwijk, \& Larkin 1998; Chakrabarty et al. 1997; Chakrabarty \& Roche 1997), that the system has an accretion disk.

Since the neutron star is currently spinning down, the radius at which the magnetosphere boundary would corotate with the disk, $r_{\text {co }}=\left(G M P^{2} / 4 \pi^{2}\right)^{1 / 3} \sim 3.6 \times 10^{4} P_{100 \mathrm{~s}}^{2 / 3} \mathrm{~km}$, where $M$ is the mass of the neutron star (assumed to be $\approx 1.4 M_{\odot}$ ) and $P_{100 \mathrm{~s}}$ is the spin period in units of $100 \mathrm{~s}$, is probably smaller than the magnetosphere radius $r_{M} \sim 4.1 \times 10^{4} L_{36}^{-2 / 7} \mathrm{~km}$, where $L_{36}$ is the $\mathrm{X}$-ray luminosity in units of $10^{36} \mathrm{ergs} \mathrm{s}^{-1}$ (Frank, King, \& Raine 1992). This value for $r_{M}$ assumes a surface magnetic field of $\sim 10^{14} \mathrm{G}$ for GX 1+4 (Makishima et al. 1988; White 1988; Chakrabarty et al. 1997; Cui 1997). Since the pulse period is $\sim 120$ s and the luminosity is typically $\lesssim 10^{37} \mathrm{ergs} \mathrm{s}^{-1}$, the period is close to the equilibrium value, for which $r_{\text {co }} \sim r_{M}$. This allows spin-down to occur even though accretion continues, the centrifugal barrier not being sufficiently effective (White 1988).

Assuming that the elliptical orbit is the correct interpretation for the origin of the modulation, the mass accretion rate (and hence the luminosity) should increase as the neutron star approaches periastron, making $r_{M}$ approach $r_{\mathrm{co}}$. As the velocity gradient between the disk material and the material flowing along the magnetic field lines decreases, the spin-down torque gets smaller and the neutron star decelerates at a slower rate. We expect that this mechanism will produce a peak in the frequency residuals close to the periastron epoch, beyond which the neutron star will start to get back to a higher spin-down rate. Occasionally, due to the highly variable mass-loss rate of the red giant, $r_{\text {co }}$ will surpass $r_{M}$ and the neutron star will spin up for a brief period of time during periastron, as observed in the BATSE frequency curve in events 5, 7, and 9. According to this picture, one would expect an increase in X-ray luminosity at periastron. Although this is only marginally indicated in the BATSE pulsed flux light curve, it should be pointed out that total flux data from the All-Sky Monitor (ASM) on board RXTE for the epoch MJD 50,088-51,044 does not correlate significantly with the BATSE pulsed flux, indicating that the pulsed flux may not be a good tracer of the accretion luminosity in this system. Furthermore, the periodic $\sim 5 \mu \mathrm{Hz}$ excursions in the residual frequency would lead to very low significance variations in the X-ray flux measured by the ASM, as we now show. Taking the fiducial torque $N_{0}=\dot{M}\left(G M_{\mathrm{X}} r_{\text {co }}\right)^{1 / 2}$ given by Bildsten et al. (1997), where $\dot{M}$ is the accretion rate and $M_{\mathrm{X}}$ is the mass of the neutron star, as an order-of-magnitude estimation (since $r_{\text {co }} \approx r_{\mathrm{M}}$ ), we can establish a lower limit to the variation in $\dot{M}(\Delta \dot{M})$ that produced the residual torque, using the fact that $\dot{\nu}=N_{0} / 2 \pi I$, where $I$ is the moment of inertia of the neutron star (Ravenhall \& Pethick 1994). Since the relative variation in flux $(F)$ scales as the relative variation in luminosity, we get $\Delta F / F \sim 0.3$ for $L \sim 10^{37} \mathrm{ergs} \mathrm{s}^{-1}$. The typical ASM GX $1+4$ flux is $\sim 1 \pm 2$ count $\mathrm{s}^{-1}$ in the $2-10 \mathrm{keV}$, so the expected variations of $\sim 0.3$ counts $\mathrm{s}^{-1}$ would be very hard to detect, given the available observational coverage. This is consistent with the lack of any significant periodic signal in our calculation of the power spectrum of the entire available ASM flux history of GX 1+4 (from MJD 50,088 to 51,353). In the elliptical orbit interpretation, one would also expect that tidal torques exerted by the red giant envelope would circularize the orbit on a short timescale (Verbunt \& Phinney 1995). However, with a period of $\sim 300$ days, the red giant radius is probably less than $7 \%$ of the binary separation, as shown below. Since the rate scales as $\left(R_{c} / a\right)^{-8}$, where $R_{c}$ is the red giant radius and $a$ is the binary separation, we do not expect the circularization timescale to be too short. Furthermore, Verbunt \& Phinney (1995) show that for orbital periods longer than about 200 days, the eccentricities of red giant binaries in open clusters span the full range.

An alternative interpretation for the observed modulation would be the presence of oscillation modes in the red giant star. For an M5 giant, persistent radial oscillations with a period of $~ 300$ days are perfectly plausible (Whitelock 1987). In this case, the oscillations could excite a modulation in the massloss rate through the stellar wind that could produce the modulated torque history. However, the stability of the infrared magnitudes of V2116 Oph (Chakrabarty \& Roche 1997) preclude it from being a long-period variable, since these stars undergo regular $\gtrsim 1 \mathrm{mag}$ variations in the infrared (Whitelock 1987). In addition, the secular optical light curve in the $R$ band obtained by our group at Laboratório Nacional de Astrofísica (Brazil) from 1991 to date shows no signs of these oscillations (Pereira et al. 1996; Pereira 1998).

It is noteworthy that the amplitude of the residual frequency oscillations in GX 1+4 cannot be attributed to Doppler shifts (Chakrabarty 1996). A firm lower limit for the companion mass is given by the X-ray mass function $f_{\mathrm{X}}(M)=$ $(c \Delta \nu / \nu)^{3} P_{\text {orb }} / 2 \pi G$, which would be equal to $\sim 210 M_{\odot}$ for a $\sim 5 \mu \mathrm{Hz}$ amplitude and a 304 day orbital period. This is clearly too massive for a red giant and actually for any stellar companion. There is also no evidence of Doppler shifts in the spectral lines of V2116 Oph (Sood et al. 1995; Chakrabarty \& 
Roche 1997), which could be an indication that the inclination of the system is fairly low.

The spectral and luminosity classification of V2116 Oph, together with the measured interstellar extinction of $A_{V} \approx 5$, is consistent with a low-mass star $\left(M \sim 0.8-2 M_{\odot}\right)$ on the firstascent red giant branch at a distance of 3-6 kpc (Chakrabarty $\&$ Roche 1997). The range of radii for such a start is $\sim 50-110$ $R_{\odot}$. The size of the Roche lobe of this object as the companion in the binary system can be estimated by the radius of a sphere with the same volume as the lobe,

$$
R_{L}=1.42 \times 10^{11} M_{\mathrm{X}}^{1 / 3} \frac{(1+q)^{1 / 3} q^{2 / 3}}{0.6 q^{2 / 3}+\ln \left(1+q^{1 / 3}\right)} P_{d} \mathrm{~cm}
$$

where $q=M_{g} / M_{\mathrm{X}}$ is the mass ratio of the red giant and the neutron star, $M_{\mathrm{X}}$ is in solar mass units, and $P_{d}$ is the orbital period in days (Eggleton 1983). Assuming $M_{\mathrm{X}}=1.4$ and $P_{d}=304$, the range of values obtained for $R_{L}$ is $546-780 R_{\odot}$ for GX $1+4$, with the binary separation ranging from 1640 to $1890 R_{\odot}$. Thus, the companion is probably not filling its Roche lobe and the accretion disk forms from the slow, dense stellar wind of the red giant.

Another interesting argument leading to a $~ 300$ day orbital period for GX $1+4$ comes from the work of van Paradijs \& McClintock (1994), according to which the absolute visual magnitudes of low-mass X-ray binary systems seem to correlate linearly with the quantity $\Sigma=P_{\text {orb }}^{2 / 3} \gamma^{1 / 2}$, where $\gamma=L_{X} / L_{\text {Edd }}$ is the accretion luminosity in units of the Eddington luminosity. Taking the value of $M_{V} \approx-4.2$ for the disk light not contaminated by $\mathrm{H} \alpha$ obtained by Jablonski \& Pereira (1997), we get $P_{\text {orb }} \sim 270 \pm 82$ days for $L_{\mathrm{X}} \sim L_{\mathrm{Edd}}$, which is fully consistent with our results. It should be noted, however, that this model is based upon the assumption that the optical emission is dominated by reprocessing of $\mathrm{X}$-rays in the accretion disk, which is not clearly the case in GX $1+4$.

In conclusion, we have shown that the long-sought orbital period of GX $1+4$ is very likely to be 304 days, as proposed in 1986 by CDD86 with marginal confidence. A more thorough covering of the X-ray luminosity of the system, with high sensitivity and spanning several cycles, will be very important for testing the elliptical orbit model.

We thank Bob Wilson from NASA/Marshall Space Flight Center for generously providing us BATSE frequency and flux data on GX $1+4$. M. P. is supported by a FAPESP Postdoctoral fellowship at INPE under grant 98/16529-9. J. B. thanks CNPq for support under grant 300689/92-6. F. J. acknowledges support by PRONEX/FINEP under grant 41.96.0908.00. We thank an anonymous referee for very important corrections and suggestions.

\section{REFERENCES}

Bildsten, L., et al. 1997, ApJS, 113, 367

Chakrabarty, D. 1996, Ph.D. thesis, Caltech

Chakrabarty, D., et al. 1997, ApJ, 481, L101

Chakrabarty, D., \& Roche, P. 1997, ApJ, 489, 254

Chakrabarty, D., van Kerkwijk, H., \& Larkin, J. E. 1998, ApJ, 497, L39

Cui, W. 1997, ApJ, 482, L163

Cutler, E. P., Dennis, B. R., \& Dolan, J. F. 1986, ApJ, 300, 551

Davidsen, A., Malina, R., \& Bowyer, S. 1977, ApJ, 211, 866

Doty, J. P., Hoffman, J. A., \& Lewin, W. H. G. 1981, ApJ, 243, 257

Eggleton, P. P. 1983, ApJ, 268, 368

Frank, J., King, A., \& Raine, D. 1992, Accretion Power in Astrophysics (2d ed.; Cambridge: Cambridge Univ. Press)

Glass, I. S., \& Feast, M. W. 1973, Nature Phys. Sci., 245, 39

Hall, R., \& Davelaar, J. 1983, IAU Circ. 3872

Jablonski, F. J., \& Pereira, M. 1997, MNRAS, 289, L17

Jablonski, F. J., Pereira, M., Braga, J., \& Gneiding, C. D. 1997, ApJ, 482, L171

Lewin, W. H. G., Ricker, G. R., \& McClintock, J. E. 1971, ApJ, 169, L17

Makishima, K., et al. 1988, Nature, 333, 746

McClintock, J. E., \& Leventhal, M. 1989, ApJ, 346, 143
Nelson, R. W., et al. 1997, ApJ, 488, L117

Pereira, M. G. 1998, Ph.D. thesis, Instituto Nacional de Pesquisas Espaciais (Brazil)

Pereira, M. G., Braga, J., \& Jablonski, F. J. 1996, Rev. Mexicana Astron. Astrofis., 4, 128

Pereira, M., et al. 1997, IAU Circ. 6749

Predehl, P., Friedrich, S., \& Staubert, R. 1995, A\&A, 294, L33

Press, W. H., Teukolsky, S. A., Vetterling, W. T., \& Flannery, B. P. 1992, Numerical Recipes in FORTRAN (2d ed.; Cambridge: Cambridge Univ. Press)

Ravenhall, D. G., \& Pethick, C. J. 1994, ApJ, 424, 846

Sakao, T., et al. 1990, MNRAS, 246, 11P

Sood, R. K., James, S. D., Lawson, W. A., Sharma, D. P., Grey, D. G., \& Manchanda, R. K. 1995, Adv. Space Res., 16(3), 131

van Paradijs, J., \& McClintock, J. E. 1994, A\&A, 290, 133

Verbunt, F., \& Phinney, E. S. 1995, A\&A, 296, 709

White, N. E. 1988, Nature, 333, 708

Whitelock, P. A. 1987, PASP, 99, 573

Wijnands, R., \& van der Klis, M. 1998, Nature, 394, 344 


\section{ERRATUM}

In the Letter "The Orbital Period of the Accreting Pulsar GX 1+4" by M. G. Pereira, J. Braga, and F. J. Jablonski (ApJ, 526, L105 [1999]), equation (2) should read

$$
R_{L}=1.42 \times 10^{11} M_{\mathrm{X}}^{1 / 3} \frac{(1+q)^{1 / 3} q^{2 / 3}}{0.6 q^{2 / 3}+\ln \left(1+q^{1 / 3}\right)} P_{d}^{2 / 3} \mathrm{~cm} .
$$

The range of values quoted for $R_{L}$ in the same paragraph in which equation (2) is inserted should read 81-116 $R_{\odot}$, with the binary separation ranging from 244 to $288 R_{\odot}$. The last statement of this paragraph should be replaced by "Thus, the companion is probably filling its Roche lobe and the accretion disk forms mainly from the stream of matter generated at the inner Lagrangian point."

In the third to the last sentence of the third paragraph of $\S 3$, the phrase "the red giant radius is probably less than $7 \%$ of the binary separation" should be revised to read "the red giant radius is probably of the order of $30 \%$ of the binary separation." 